\title{
Use of a fully covered metal stent to treat obstruc- tion of the minor papilla in pancreas divisum
}

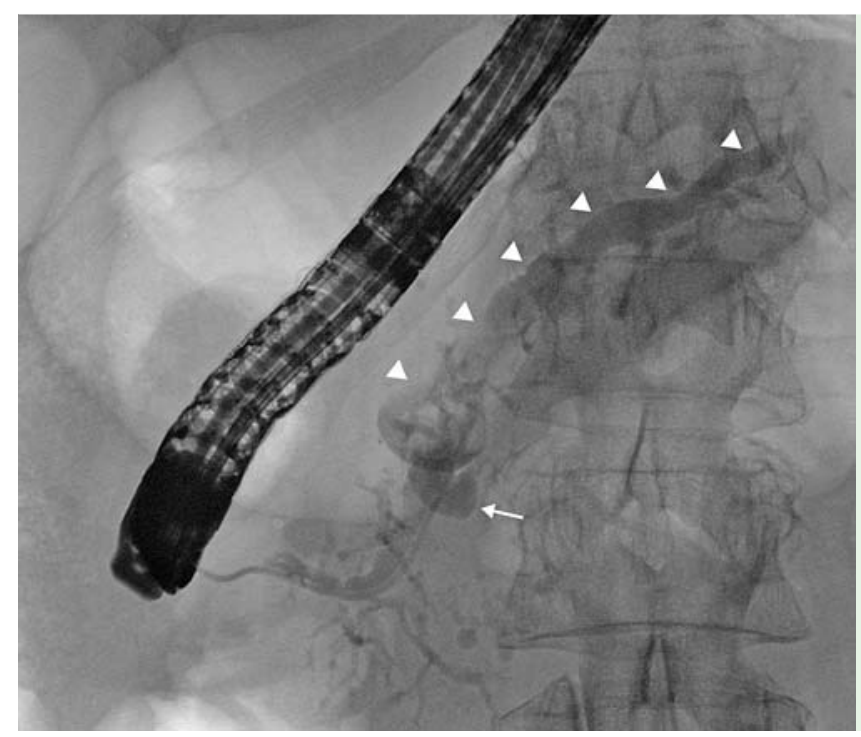

Fig. 1 Endoscopic retrograde pancreatography using cannulation of the minor papilla. The dilated pancreatic duct (arrow heads) with impacted stone (arrow) can be seen.

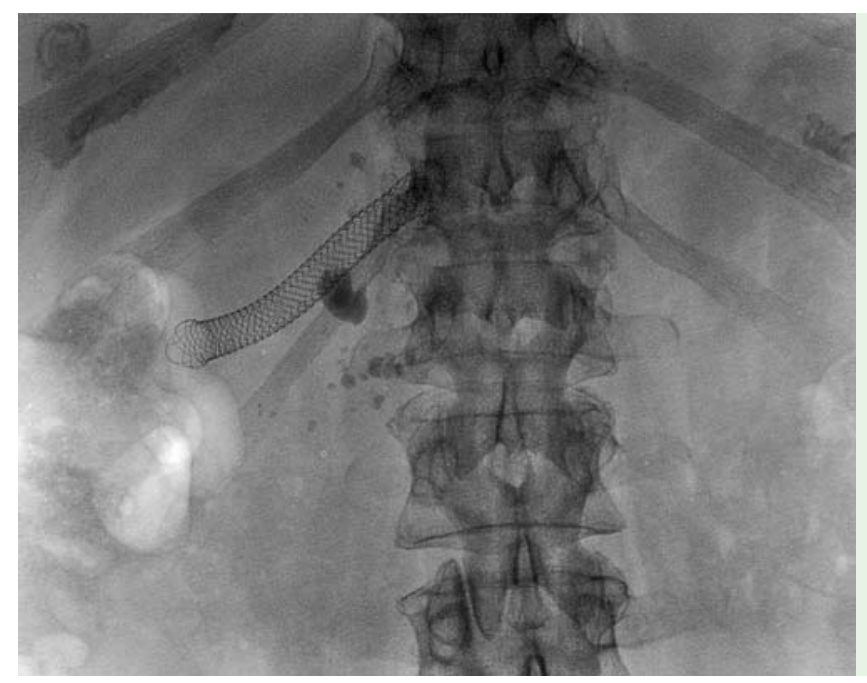

Fig. 2 Plain radiographic image of the pancreatic region, showing the pancreatic duct stone and fully covered self-expanding metal stent 5 months after insertion through the minor papilla.

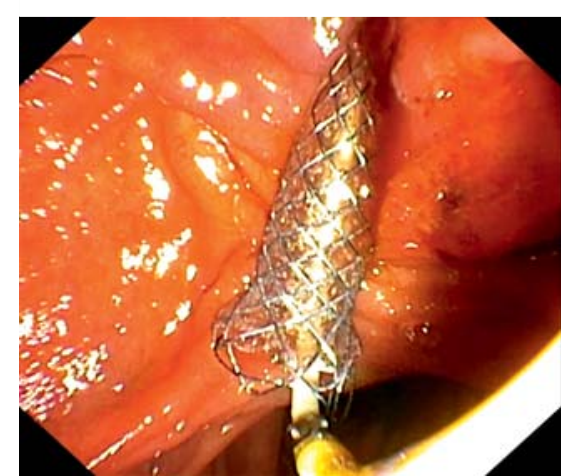

Fig. 3 The fully covered self-expanding metal stent through the minor papilla.

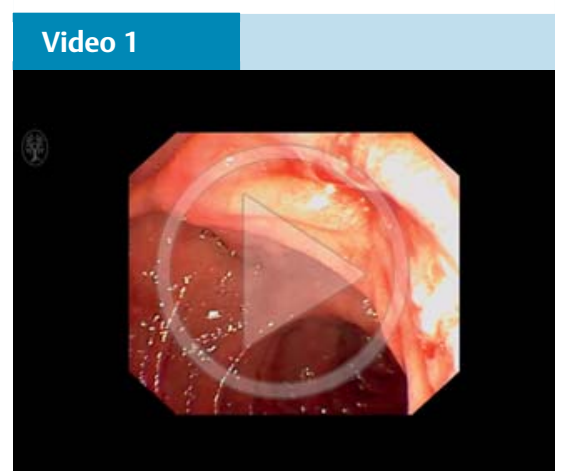

Introduction and release of the fully covered self-expanding metal stent through the minor papilla, and visualization of the stent lumen after release.
A 70-year-old woman was admitted with an episode of epigastric pain radiating into her back, accompanied by intermittent nausea and vomiting. Acute pancreatitis was diagnosed and transabdominal ultrasound revealed a dilated pancreatic duct of $6 \mathrm{~mm}$ due a ductal stone.

The first attempt of endoscopic retrograde pancreatography (ERP) failed to cannulate the pancreatic duct. Following a magnetic resonance cholangiopancreatography, the patient was diagnosed with a complete pancreas divisum. Hence, the pancreatic duct was accessed via the minor papilla, and ERP showed a dilated duct with an impacted stone near the orifice ( $\bullet$ Fig. 1 ). Unfortunately, the stone could not be removed despite dilation of the distal duct. Surgical interventions or shock wave lithotripsy were discussed, but the patient preferred an endoscopic approach. Subsequently, a fully covered self-expanding metal stent (SEMS, WallFlex $8 \times 60 \mathrm{~mm}$; Boston Scientific Corp., Marlborough, Massachusetts, USA) was placed for 5 months in the minor papilla ( Fig. 2). After implantation of the SEMS, the patient had no complaints and reported fewer episodes of epigastric pain.

During pancreaticoscopy with electrohydraulic lithotripsy, after retrieval of the SEMS only fragments of the stone could be removed. Because of the good response following implantation of the covered SEMS, we again decided to place an SEMS (๑ Fig. 3, Video 1). So far, 14 months after the initial stent implantation, the patient has gained weight and, apart from occasional episodes of pain, she reports no complaints.

Our results are in line with a case series reported by Liao et al., which showed similar success in three patients [1]; however, in these cases the SEMS passed spontaneously after 6 months, and data on longer follow-up after migration are missing. In conclusion, this case describes the possibility of draining obstruction due to pancreatic ductal stone in the minor papilla in complete pancreas divisum using a fully covered SEMS, with good clinical response.

\section{Endoscopy_UCTN_Code_TTT_1AR_2AZ}

Competing interests: None

Alexander Hann, Benjamin M. Walter, Alexander Meining

Department of Internal Medicine I, Ulm University, Ulm, Germany 


\section{Reference}

1 Liao Z, Li ZS, Wang W et al. Endoscopic placement of a covered self-expandable metal stent in the minor papilla in patients with chronic pancreatitis and pancreas divisum. Endoscopy 2009; 41 (Suppl. 02): E302 - 303
Bibliography

DOI http://dx.doi.org/

10.1055/s-0042-119048

Endoscopy 2016; 48: E390-E391

(c) Georg Thieme Verlag KG

Stuttgart · New York

ISSN 0013-726X
Corresponding author

Alexander Hann, MD

Uniklinik Ulm

Klinik für Innere Medizin I

Albert-Einstein-Allee 23

89081 Ulm

Fax: +49-731-50044502

alexander.hann@uniklinik-ulm.de 